\title{
Luttinger liquids and composite fermions in nanostructures: what is the nature of the edge states in the fractional quantum Hall regime?
}

\author{
Michael R. Geller, Daniel Loss, George Kirczenow \\ Department of Physics, Simon Fraser University, Burnaby B.C. V5A 1S6, Canada
}

(Received 20 May 1996)

\begin{abstract}
We study the Aharonov-Bohm conductance oscillations of a constriction with an antidot in the fractional quantum Hall regime using a recently proposed composite-fermion Fermi liquid theory, and also using Wen's chiral Luttinger liquid theory extended to include mesoscopic effects. The predictions of the composite-fermion Fermi liquid theory are very similar to standard Fermi liquid theory and are consistent with recent experiments. In our chiral Luttinger liquid theory, which is valid in an experimentally realizable 'strongantidot-coupling' regime for bulk filling factors $g=1 / q$ ( $q$ odd), the finite size of the antidot introduces a new temperature scale $T_{0} \equiv \hbar v / \pi k_{B} L$, where $v$ is the Fermi velocity and $L$ is the circumference of the antidot edge state. Chiral Luttinger liquid theory predicts the low-temperature $\left(T \ll T_{0}\right)$ Aharonov-Bohm amplitude to vanish with temperature as $T^{2 q-2}$, in striking contrast to Fermi liquid theory $(q=1)$. Near $T \approx T_{0}$, there is a pronounced maximum in the amplitude, also in contrast to a Fermi liquid. At high temperatures $\left(T \gg T_{0}\right)$, however, we predict a new crossover to a $T^{2 q-1} e^{-q T / T_{0}}$ temperature dependence, which is qualitatively similar to Fermi liquid behavior. We show how measurements in the strong-antidot-coupling regime, where transmission through the device is weak, should be able to distinguish between Fermi liquid and chiral Luttinger liquid behavior both at low and high temperatures and in the linear and nonlinear response regimes. Finally, we predict new mesoscopic edge-current oscillations, which are similar to persistent current oscillations in a mesoscopic ring, except that they are not reduced in amplitude by disorder. In the fractional regime, these 'chiral persistent currents' have a universal non-Fermi-liquid temperature dependence, and may be another ideal system to observe a chiral Luttinger liquid.
\end{abstract}

(c) 1997 Academic Press Limited

Key words: chiral Luttinger liquids, Aharonov-Bohm effect, composite fermions.

\section{Introduction}

It is well-known that the integer quantum Hall effect [1] and many related phenomena that are observed in nanoscale semiconductor devices [2] can be understood in terms of Fermi liquid (FL) theories of magnetic edge-state transport [3] such as the Büttiker-Landauer formalism [4]. However, as was shown by Laughlin [5], the fractional quantum Hall effect (FQHE) [6] occurs because electron-electron interactions result in the formation of incompressible states of two-dimensional electron systems at special filling fractions of 

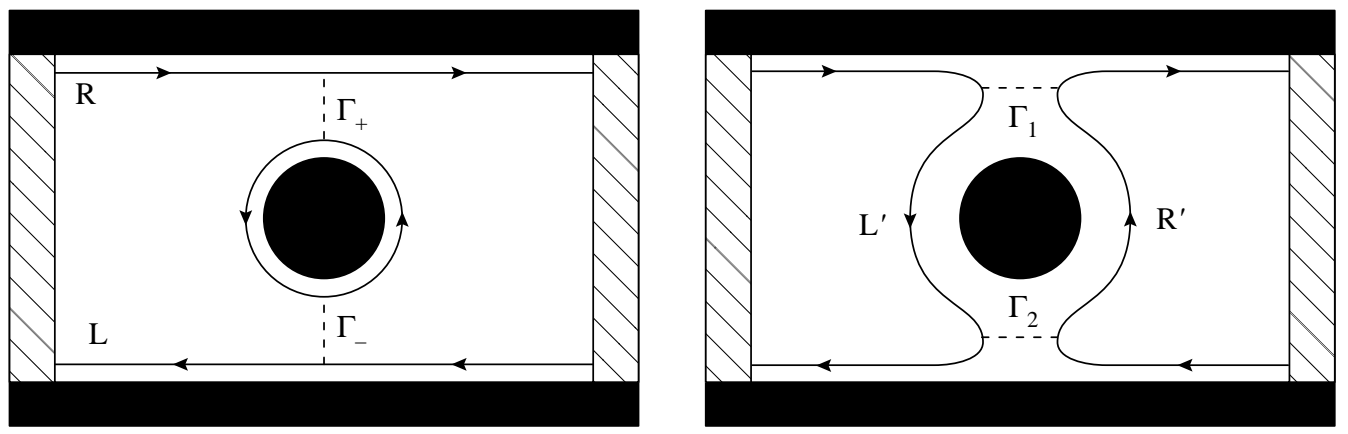

Fig. 1. Aharonov-Bohm effect geometry in the (A) weak-antidot-coupling and (B) strong-antidot-coupling regimes. In both cases the dashed lines represent weak tunneling processes.

a Landau level. Thus, Wen [7] and others [8-11] have proposed and developed a chiral Luttinger liquid (CLL) theory of edge states in the FQHE regime, in which electron-electron interactions play a crucial role and whose predictions can differ dramatically from those of FL theory. On the other hand, as was pointed out by Jain [12], the incompressible many-body states that give rise to the FQHE can be thought of, in a mean field sense, as arising from the spectral gaps between the single-particle Landau levels of quasiparticles known as 'composite fermions.' Recently a composite-fermion edge state theory of transport has been proposed [13] which also adopts this single-particle view of the FQHE. It predicts the properties of edge states in the FQHE regime to be close to-but not identical with-those of a conventional FL.

Experiments on semiconductor nanostructures should be able to distinguish between CLL and FL theories of fractional edge states. The first experiment of this kind was performed by Milliken et al. [14] on a device with a disordered constriction. The results obtained were consistent with the predictions of CLL. More recent experiments by Franklin et al. [15] on Aharonov-Bohm (AB) oscillations and by Maasilta and Goldman [16] on resonant tunneling in closely related but cleaner devices-constrictions containing an antidot (see Fig. 1) - have yielded evidence that is consistent with the FL picture of FQHE edge states. On the other hand, Chang et al. [17] working with an entirely different type of device have very recently reported behavior consistent with CLL edge state theory. Thus the nature of FQHE edge states remains an open problem both experimentally and theoretically.

In this paper we address the problem of $\mathrm{AB}$ conductance oscillations of constrictions with an antidot in the FQHE regime theoretically, from both the composite-fermion FL and CLL points of view. We first outline the treatment of these resonances in the integer regime based on the Büttiker-Landauer FL approach, and then describe the salient results of the composite-fermion FL theory in the fractional regime. We then develop a CLL theory of the $\mathrm{AB}$ oscillations for the case of weak transmission through the constriction containing the antidot (our 'strong-antidot-coupling' regime), compare the results obtained with those of FL theory, and we propose experiments that should be able to distinguish definitively between CLL and FL fractional edge states in the strong-coupling regime. Our CLL theory also applies to the case of weak-antidot-coupling for values of AB flux which are off resonance [18]. The CLL theory we present assumes that there is only one relevant FQHE edge state present.

Finally, we study the response of an edge state to an additional AB flux. We find new mesoscopic edgecurrent oscillations, which are similar to persistent current oscillations in a mesoscopic ring, except that they are not reduced in amplitude by disorder. In the FQHE regime, these 'chiral persistent currents' have a universal non-Fermi-liquid temperature dependence and may be another ideal system to observe a chiral Luttinger liquid. 


\section{Büttiker-Landauer fermi liquid theory}

The system of an antidot in a constriction in the integer regime has been studied extensively both experimentally [19] and theoretically [20]. The theoretical work has been based on Büttiker-Landauer edge state theory, which is valid for noninteracting electrons. At finite temperatures the net current $I$ flowing from drain to source through the constriction with the antidot is

$$
I=-\frac{e}{h} \int d \epsilon \hat{T}(\epsilon)\left[n_{\mathrm{F}}\left(\epsilon-\mu_{\mathrm{s}}\right)-n_{\mathrm{F}}\left(\epsilon-\mu_{\mathrm{d}}\right)\right]
$$

where $\hat{T} \equiv \operatorname{Tr} t t^{\dagger}$ is the multichannel edge-state transmission probability of the constriction with the antidot, which depends on the energy $\epsilon$. Here $n_{\mathrm{F}}(\epsilon) \equiv\left(e^{\epsilon / T}+1\right)^{-1}$ is the Fermi distribution function, with $T$ being the temperature, and $\mu_{\mathrm{s}}$ and $\mu_{\mathrm{d}}$ are the electrochemical potentials of the source and drain that feed electrons via edge states to the constriction. In linear response, this reduces to a two-terminal conductance for the device given by

$$
G_{\mathrm{LR}}=-\frac{e^{2}}{h} \int d \epsilon \frac{\partial n_{\mathrm{F}}}{\partial \epsilon} \hat{T}(\epsilon)
$$

The transmission probability $\hat{T}(\epsilon)$ has been evaluated for a variety of edge state configurations in Ref. [20] using unitary edge-state scattering matrices. For the case shown in Fig. 1, it takes the form

$$
\hat{T}=1-\frac{\left(1-T_{A}\right)^{2}}{T_{A}^{2}-2 T_{A} \cos \Omega+1},
$$

where $T_{A}$ is the probability that an electron in an edge state is transmitted directly through the constriction on either side of the antidot without being scattered out of that edge state, and is assumed to be the same for the edge states on either side of the antidot. In the notation of Fig. $1 \mathrm{~A}, T_{A}=1-\left|\Gamma_{ \pm}\right|^{2} . \Omega$ is the phase accumulated by an electron in a complete orbit of the antidot; up to an additive constant it is approximately given by $2 \pi \Phi / \Phi_{0}+\epsilon L / v$, where $\Phi$ is the $\mathrm{AB}$ flux, $\Phi_{0} \equiv h c / e$ is the flux quantum, $L$ is the antidot circumference, and $\epsilon$ is the electron energy. In the limit of small transmission probability $T_{A}$ we have

$$
\hat{T} \approx 2 T_{A}(1-\cos \Omega) .
$$

This limit corresponds to the strong-antidot-coupling case for which the CLL theory described in this article has been developed. We will be comparing the predictions of the above Büttiker-Landauer FL theory with those of our CLL theory below.

\section{Temperature dependence of the Aharonov-Bohm oscillations in the composite-fermion picture}

A detailed treatment of the composite-fermion FL theory of antidot AB resonances will be presented elsewhere [21]. Here we will outline the main results of the theory of the temperature dependence of the composite-fermion conductance resonances, since this has been the aspect of interest in the experimental studies that have sought to distinguish between the FL and CLL theories of fractional edge states. In this section we use the term Fermi liquid to apply to interacting electron systems that may be treated as weakly interacting or noninteracting quasiparticle systems. The composite fermion edge state energy level structure differs qualitatively from that of ordinary Landau levels in the integer regime because of the presence of a densitydependent contribution to the effective magnetic field experienced by the composite fermions [12, 13, 2123]. Furthermore, the effective electrochemical potentials of the composite fermions differ in general from those of the corresponding electrons because the composite fermions experience a fictitious electric field not experienced by electrons $[13,21,24]$. Nevertheless, expressions for the conductance of composite fermion 
systems have been obtained in terms of multichannel transmission and reflection probabilities for composite fermions [21]. If the effective magnetic field experienced by the composite fermions is parallel or antiparallel to the true magnetic field in the bulk and parallel to the true magnetic field at the centers of the channels between the antidot and the surrounding gates (as in the experiments of Franklin et al. [15] and of Maasilta and Goldman [16]), the net composite fermion current through the device takes the form

$$
I=-\frac{e}{h} \int d \epsilon \hat{T}^{*}(\epsilon)\left[n_{\mathrm{F}}\left(\epsilon-\mu_{\mathrm{s}}^{*}\right)-n_{\mathrm{F}}\left(\epsilon-\mu_{\mathrm{d}}^{*}\right)\right]
$$

Since the composite fermion edge states are taken to be a Fermi liquid, eqn (5) closely resembles (1), however the multichannel transmission probability $\hat{T}^{*}$ and the electrochemical potentials $\mu_{\mathrm{s}}^{*}$ and $\mu_{\mathrm{d}}^{*}$ that appear are those of composite fermions, not electrons. The two-terminal conductance of the constriction with the antidot is then given in linear response by

$$
G_{C F}=\frac{e^{2}}{h} \cdot \frac{-\int d \epsilon \partial n_{\mathrm{F}} / \partial \epsilon \hat{T}^{*}}{1-m \int d \epsilon \partial n_{\mathrm{F}} / \partial \epsilon \hat{T}^{*}}
$$

where $m$ is the number of quanta of fictitious magnetic flux carried by each composite fermion [25]. The form of this expression differs from the Büttiker-Landauer linear response expression (2), although both have been derived for Fermi liquid systems; the difference between the two forms arises from the presence of the fictitious electric field in the composite fermion theory. However, the shapes of the resonances given by the two expressions turn out to be very similar [21] since $\alpha /(1+m \alpha)$ is a monotonically and smoothly increasing function of $\alpha$ and is nearly linear over the small ranges of $\alpha$ that are of interest in practice. Furthermore, although $G_{C F}$ does not obey the standard FL linear response relation

$$
G_{\mathrm{T}}=-\int d \epsilon G_{0}(\epsilon) \frac{\partial n_{\mathrm{F}}}{\partial \epsilon},
$$

between the zero temperature conductance $G_{0}(\epsilon)$ at energy $\epsilon$ and the finite temperature conductance $G_{T}$ exactly, the discrepancy turns out to be very small in practice again because of the properties of the mapping $\alpha \rightarrow \alpha /(1+m \alpha)$. Thus the temperature dependence of the $\mathrm{AB}$ resonances predicted by the composite-fermion FL theory is very close to that predicted by standard FL phenomenology as reflected in eqn (7). In particular, if in addition $\hat{T}^{*}$ is of the form of eqn (4) then the functional temperature dependence of FL theory and composite fermion FL theory is well described by eqn (29) below. In fact the experimental data of Franklin et al. were analyzed with eqn (29) [26]. The experiments [15, 16] that have recently reported FL behavior of the $\mathrm{AB}$ resonances have not been sensitive enough to detect the difference between composite-fermion FL and standard FL theory.

Although the above composite-fermion FL theory is consistent with the experiments, this does not in itself rule out the possibility that the fractional edge states may be Luttinger liquids because no detailed CLL theory of the $\mathrm{AB}$ effect has been available. A Luttinger liquid theory of antidot $\mathrm{AB}$ oscillations, that is applicable in a regime different from the one in which the experiments of Franklin et al. [15] and of Maasilta and Goldman [16] were carried out, is presented below.

\section{Finite-size chiral Luttinger liquid with topological excitations}

To study mesoscopic effects associated with edge states in the FQHE, we shall extend chiral Luttinger liquid theory to include finite-size effects. Finite-size effects in nonchiral Luttinger liquids have been discussed by Haldane [27] and by Loss [28]. To proceed in the chiral case we bosonize the electron field operators $\psi_{ \pm}(x)$ according to the convention

$$
\rho_{ \pm}= \pm \frac{\partial_{x} \phi_{ \pm}}{2 \pi}
$$


where $\rho_{ \pm}(x)$ is the normal-ordered charge density and $\phi_{ \pm}$is a chiral scalar field for right $(+)$or left $(-)$ movers. The dynamics of $\phi_{ \pm}$is governed by Wen's Euclidian action [7]

$$
S_{ \pm}=\frac{1}{4 \pi g} \int_{0}^{L} d x \int_{0}^{\beta} d \tau \partial_{x} \phi_{ \pm}\left( \pm i \partial_{\tau} \phi_{ \pm}+v \partial_{x} \phi_{ \pm}\right)
$$

where $g=1 / q$ ( $q$ odd) is the bulk filling factor and $v$ is the Fermi velocity. Here $L$ is the size (i.e. length) of a given edge state. When $q=1$, eqn (9) describes noninteracting chiral electrons. The field theory described by eqn (9) may be canonically quantized by imposing the equal-time commutation relation (modulo periodic extension)

$$
\left[\phi_{ \pm}(x), \phi_{ \pm}\left(x^{\prime}\right)\right]= \pm i \pi g \operatorname{sgn}\left(x-x^{\prime}\right) .
$$

We then decompose $\phi_{ \pm}$into a nonzero-mode part $\bar{\phi}_{ \pm}(x)$ satisfying periodic boundary conditions that describes the neutral excitations, and a zero-mode part $\phi_{ \pm}^{0}$ that describes charged excitations: $\phi_{ \pm}=\bar{\phi}_{ \pm}+\phi_{ \pm}^{0}$. The nonzero-mode part may be expanded in a basis of Bose annihilation and creation operators in the usual way,

$$
\bar{\phi}_{ \pm}(x)=\sum_{k \neq 0} \theta( \pm k) \sqrt{\frac{2 \pi g}{|k| L}}\left(a_{k} e^{i k x}+a_{k}^{\dagger} e^{-i k x}\right) e^{-|k| a / 2},
$$

with coefficients determined by the requirement that $\bar{\phi}_{ \pm}$itself satisfies (10) in the $L \rightarrow \infty$ limit. In a finite-size system, however,

$$
\left[\bar{\phi}_{ \pm}(x), \bar{\phi}_{ \pm}\left(x^{\prime}\right)\right]= \pm i \pi g \operatorname{sgn}\left(x-x^{\prime}\right) \mp 2 \pi i g\left(x-x^{\prime}\right) / L
$$

so we must require the zero-mode part to satisfy

$$
\left[\phi_{ \pm}^{0}(x), \phi_{ \pm}^{0}\left(x^{\prime}\right)\right]= \pm 2 \pi i g\left(x-x^{\prime}\right) / L
$$

for the total field to satisfy eqn (10). An expansion analogous to eqn (11) for $\phi_{ \pm}^{0}$ may be constructed from the condition (13) and, in addition, the requirement

$$
\phi_{ \pm}^{0}(x+L)-\phi_{ \pm}^{0}(x)= \pm 2 \pi N_{ \pm},
$$

which follows from eqn (8), where $N_{ \pm} \equiv \int_{0}^{L} d x \rho_{ \pm}$is the charge of an excited state relative to the ground state. Conditions (13) and (14) together determine $\phi_{ \pm}^{0}$, up to an additive $c$-number constant, as

$$
\phi_{ \pm}^{0}(x)= \pm \frac{2 \pi}{L} N_{ \pm} x-g \chi_{ \pm}
$$

where $\chi_{ \pm}$is a phase operator conjugate to $N_{ \pm},\left[\chi_{ \pm}, N_{ \pm}\right]=i$. Equations (11) and (15) may now be used to write the (normal-ordered) Hamiltonian corresponding to (9) as

$$
H_{ \pm}=\frac{v}{4 \pi g} \int_{0}^{L} d x\left(\partial_{x} \phi_{ \pm}\right)^{2}=\frac{\pi v}{g L} N_{ \pm}^{2}+\sum_{k \neq 0} \theta( \pm k) v|k| a_{k}^{\dagger} a_{k} .
$$

In a finite-size system, the level spacing for neutral and charged excitations scale with system size as $1 / L$ and become gapless in the $L \rightarrow \infty$ limit.

What are the allowed eigenvalues of $N_{ \pm}$? The answer may be determined by bosonization: To create an electron, we need a $\pm 2 \pi$ kink in $\phi_{ \pm}$. The electron field operators can therefore be bosonized as

$$
\psi_{ \pm}(x)=\frac{1}{\sqrt{2 \pi a}} e^{i\left[\phi_{ \pm}(x) \pm \pi x / L\right] / g},
$$

where $a$ is the same microscopic cut-off length that appears in eqn (11). The additional c-number phase factor is chosen for convenience. To see that (17) is valid, note that $\left[\rho_{ \pm}(x), \psi_{ \pm}^{\dagger}\left(x^{\prime}\right)\right]=\delta\left(x-x^{\prime}\right) \psi_{ \pm}^{\dagger}\left(x^{\prime}\right)$, so $\psi_{ \pm}^{\dagger}(x)$ 
creates an electron at position $x$. Equation (17) implies that $\psi_{ \pm}(x+L)=\psi_{ \pm}(x) e^{ \pm i 2 \pi N_{ \pm} / g}$. Thus, periodic boundary conditions on $\psi_{ \pm}(x)$ lead to the important result that the allowed eigenvalues of $N_{ \pm}$are given by

$$
N_{ \pm}=n g,
$$

which means that there exists fractionally charged excitations or quasiparticles, as expected in a FQHE system.

Finally, we note that coupling to an $\mathrm{AB}$ flux $\Phi$ is achieved by adding $\delta \mathcal{L}=\frac{1}{c} j_{ \pm} A$ to the Lagrangian, where $j_{ \pm}= \pm \frac{e}{2 \pi} \partial_{t} \phi_{ \pm}$is the bosonized current density and $A$ is a vector potential. The flux couples only to the zero-modes, and results in the replacement $N_{ \pm}^{2} \rightarrow\left(N_{ \pm} \pm g \Phi / \Phi_{0}\right)^{2}$ in (16), where $\Phi_{0} \equiv h c / e$.

\section{Scaling theory}

We begin our study of the $\mathrm{AB}$ effect by performing a perturbative renormalization group (RG) analysis in the weak-antidot-coupling regime. In this case $S=S_{0}+\delta S$, where $S_{0} \equiv S_{L}+S_{R}+S_{A}$ is the sum of actions of the form (9) for the left moving, right moving, and antidot edge states, respectively, and $\delta S \equiv$ $\sum_{m} \int_{\tau}\left(V_{+}+V_{-}+\right.$c.c. $)$is the weak coupling between them. Here

$$
V_{ \pm}(\tau) \equiv \frac{\Gamma_{ \pm}^{(m)}}{2 \pi a} e^{i m \phi_{ \pm}\left(x_{ \pm}, \tau\right)} e^{-i m \phi_{A}\left(x_{ \pm}, \tau\right)}
$$

describes the tunneling of $m$ quasiparticles from an incident edge state into the antidot edge state at point $x_{ \pm}$ with dimensionless amplitude $\Gamma_{ \pm}^{(m)}$ (see Fig. 1A) [29]. We assume the leads, described by $S_{L}$ and $S_{R}$, to be macroscopic, and we also assume for simplicity that $\left|\Gamma_{-}^{(m)}\right|=\left|\Gamma_{+}^{(m)}\right|$. We shall need the correlation function $C_{ \pm}(x, \tau) \equiv\left\langle e^{i m \phi_{ \pm}(x, \tau)} e^{-i m \phi_{ \pm}(0)}\right\rangle$, which, at zero temperature and for values of $x$ such that $x \ll L$, is given by

$$
C_{ \pm}(x, \tau)=\left(\frac{i a}{ \pm x+i v \tau+i a}\right)^{2 \Delta_{ \pm}}
$$

where $\Delta_{ \pm}=m^{2} g / 2$ is the scaling dimension of $e^{i m \phi_{ \pm}}$.

Consider now the correlation function

$$
\left\langle V_{+}^{\dagger}(\tau) V_{+}(0)\right\rangle=\frac{\left|\Gamma_{+}^{(m)}\right|^{2}}{4 \pi^{2} a^{2}}\left\langle e^{-i m \phi_{+}\left(x_{+}, \tau\right)} e^{i m \phi_{+}\left(x_{+}, 0\right)}\right\rangle\left\langle e^{i m \phi_{A}\left(x_{+}, \tau\right)} e^{-i m \phi_{A}\left(x_{+}, 0\right)}\right\rangle,
$$

which arises in a perturbative calculation of the partition function $Z=\int \mathcal{D} \phi_{L} \mathcal{D} \phi_{R} \mathcal{D} \phi_{A} e^{-S}$. For $\left\langle V_{+}^{\dagger}(\tau) V_{+}(0)\right\rangle$ and therefore $Z$ to be invariant under a small decrease in cut-off $a \rightarrow a^{\prime}=s a$ we need $\Gamma^{\prime}=s^{1-2 \Delta} \Gamma$, or

$$
\frac{d \Gamma_{+}^{(m)}}{d \ell}=\left(1-m^{2} g\right) \Gamma_{+}^{(m)},
$$

where $\ell \equiv \ln \left(a / a^{\prime}\right) . \Gamma_{-}^{(m)}$ satisfies an identical flow equation. These flow equations, which show that quasiparticle $(m=1)$ backscattering processes are relevant and electron $(m=1 / g)$ backscattering is irrelevant when $g=1 / 3$, were first derived by Kane and Fisher [29] using momentum-shell RG.

Next consider the correlation function $\left\langle V_{+}^{\dagger}(\tau) V_{+}\left(\tau^{\prime}\right) V_{-}^{\dagger}\left(\tau^{\prime \prime}\right) V_{-}(0)\right\rangle$. A Wick expansion gives local terms as in (21), and, in addition, nonlocal antidot terms like $\left\langle e^{i m \phi_{A}(x, \tau)} e^{-i m \phi_{A}(0)}\right\rangle$ with $x \neq 0$. Equation (20) shows that the nonlocal terms (for $x \ll L$, with $L$ now the size of the antidot edge state) scale the same way as the local terms. The Kane-Fisher flow equations (22) are therefore valid in the antidot problem considered here.

\section{Aharonov-Bohm effect in the strong-antidot-coupling regime}

The RG analysis of the previous section shows that off resonance [18] and at low temperatures the antidot will be in the strongly coupled regime shown in Fig. 1B. Furthermore, if the antidot system starts in the strongly coupled regime, it will stay in this regime throughout the ranges of temperature and magnetic field important 
experimentally, because the quasiparticle backscattering process (which would be relevant in the RG sense) is not allowed in this edge-state configuration and only electrons can tunnel [30]. The strong-antidot-coupling regime therefore admits a perturbative treatment [30], to which we now turn. Details of the calculations shall be given elsewhere.

The current $I$ passing between edge states $L^{\prime}$ and $R^{\prime}$ as a function of their potential difference $V$ may be calculated for small tunneling amplitudes $\Gamma_{i}(i=1,2)$, which for simplicity are taken to be equal apart from $\mathrm{AB}$ phase factors [31]. The result is

$$
I=-2|\Gamma|^{2} \operatorname{Im}\left[X_{11}(\omega)+X_{22}(\omega)+e^{i\left(2 \pi \Phi / \Phi_{0}\right)} X_{12}(\omega)+e^{-i\left(2 \pi \Phi / \Phi_{0}\right)} X_{21}(\omega)\right]_{\omega=V},
$$

where $X_{i j}(\omega)$ is the Fourier transform of $X_{i j}(t) \equiv-i \theta(t)\left\langle\left[B_{i}(t), B_{j}^{\dagger}(0)\right]\right\rangle$ and $B_{i} \equiv \psi_{L}\left(x_{i}\right) \psi_{R}^{\dagger}\left(x_{i}\right)$ is an electron tunneling operator acting at point $x_{i}$. This response function can be calculated using bosonization techniques and the result for filling factor $g=1 / q$ is

$$
X_{i j}(t)=-\theta(t) \frac{a^{2 q-2}}{2 \pi^{2}} \operatorname{Im} \frac{\left(\pi / L_{\mathrm{T}}\right)^{2 q}}{\sinh ^{q}\left[\pi\left(x_{i}-x_{j}+v t+i a\right) / L_{T}\right] \sinh ^{q}\left[\pi\left(x_{i}-x_{j}-v t-i a\right) / L_{T}\right]},
$$

where $L_{T} \equiv v / T$ is the thermal length. When $q=1,(24)$ is the response function for free chiral electrons. Each term $X_{i j}$ in (23) corresponds to a process occurring with a probability proportional to $\left|\Gamma_{i} \Gamma_{j}\right|$. The local terms $X_{11}$ and $X_{22}$ therefore describe independent tunneling at $x_{1}$ and $x_{2}$, respectively, whereas the nonlocal terms $X_{12}$ and $X_{21}$ describe coherent tunneling through both antidot constrictions. The AB phase naturally couples only to the latter. We shall see that the local contributions behave exactly like the tunneling current in a quantum point contact. The $\mathrm{AB}$ effect, however, is a consequence of the nonlocal terms, and we shall show that there are new non-Fermi-liquid phenomena associated with these terms that are directly accessible to experiment.

We have Fourier transformed (24) exactly and find a crossover behavior in the nonlocal response functions when the thermal length $L_{T}$ becomes less than $\left|x_{i}-x_{j}\right|$. The finite size of the antidot therefore provides an important new temperature scale (with units now restored)

$$
T_{0} \equiv \frac{\hbar v}{\pi k_{B} L},
$$

where $L$ is the circumference of the edge state around the antidot. For example, a Fermi velocity $v$ of $10^{6} \mathrm{~cm} \mathrm{~s}^{-1}$ and circumference $L$ of $1 \mu \mathrm{m}$ yields $T_{0} \approx 20 \mathrm{mK}$. Note that $T_{0}$ is closely related to the energy level spacing $\Delta E \equiv 2 \pi v / L$ for noninteracting electrons with linear dispersion in a ring of circumference $L$ : $T_{0}=\Delta E / 2 \pi^{2}$.

The current can generally be written as

$$
I=I_{0}+I_{A B} \cos \left(2 \pi \Phi / \Phi_{0}\right),
$$

where $I_{0}$ is the direct current resulting from the local terms and $I_{A B}$ is the $\mathrm{AB}$ current resulting from the nonlocal terms.

For noninteracting electrons, the Büttiker-Landauer formula or our perturbation theory with $q=1$ may be used to show that $I^{F L}=I_{0}^{F L}+I_{A B}^{F L} \cos \left(2 \pi \Phi / \Phi_{0}\right)$, where

$$
I_{0}^{F L}=|t|^{2} V
$$

is the direct contribution, and the $\mathrm{AB}$ part is given by

$$
I_{\mathrm{AB}}^{F L}=|t|^{2} \frac{2 \pi k_{B} T}{\sinh \left(T / T_{0}\right)} \sin (V L / 2 v),
$$

where $|t|^{2}$ is the source-to-drain transmission probability [31]. 
In the linear response regime we generally write $I=G V$, where, for a Fermi liquid, $G_{0}^{\mathrm{FL}}=|t|^{2}$, and

$$
G_{\mathrm{AB}}^{\mathrm{FL}}=|t|^{2} \frac{T / T_{0}}{\sinh \left(T / T_{0}\right)} .
$$

The exact current-voltage relation for the $g=1 / 3$ chiral Luttinger liquid is

$$
I_{0}=|\Gamma|^{2} \frac{a^{4} V}{120 \pi v^{6}}\left[64 \pi^{4} T^{4}+20 V^{2} \pi^{2} T^{2}+V^{4}\right],
$$

and

$$
\begin{aligned}
I_{\mathrm{AB}}=-|\Gamma|^{2} \frac{a^{4} \pi^{2}}{v^{6}} \frac{T^{3}}{\sinh ^{3}\left(T / T_{0}\right)}\{ & \sin \left(\frac{V L}{2 v}\right) \cdot\left[V^{2}+4 \pi^{2} T^{2}\left(1-3 \operatorname{coth}^{2}\left(\frac{T}{T_{0}}\right)\right)\right] \\
& \left.+6 \pi V T \cos \left(\frac{V L}{2 v}\right) \operatorname{coth}\left(\frac{T}{T_{0}}\right)\right\} .
\end{aligned}
$$

In the limit $L \rightarrow 0, I_{\mathrm{AB}}$ always reduces to $I_{0}$. To see this in the $g=1 / 3$ case one has to expand the terms in the bracket of eqn (31) up to third order in $L$ (all lower orders cancel exactly).

We now summarize our results for general $q$. We shall for convenience summarize the transport properties as a function of temperature for fixed voltage, first for $V \ll T_{0}$ and then for $V \gg T_{0}$.

\subsection{Low voltage regime: $V \ll T_{0}$}

In this case there are three temperature regimes. When $T \ll V \ll T_{0}$, both $I_{0}$ and $I_{A B}$ have nonlinear behavior, varying with voltage as $V^{2 q-1}$.

When the temperature exceeds $V$, the response becomes linear. When $V \ll T \ll T_{0}$, both $G_{0}$ and $G_{A B}$ vary with temperature as

$$
G \propto\left(\frac{T}{T_{F}}\right)^{2 q-2}
$$

where $T_{\mathrm{F}} \equiv v / a$ is an effective Fermi temperature.

For example, when $q=3$, the exact conductance [obtained from eqn (31)] is

$$
G_{\mathrm{AB}} \propto \frac{\left(T / T_{\mathrm{F}}\right)^{4}}{\sinh ^{3}\left(T / T_{0}\right)}\left\{3 \operatorname{coth}\left(\frac{T}{T_{0}}\right)+\left(\frac{T}{T_{0}}\right)\left[1-3 \operatorname{coth}^{2}\left(\frac{T}{T_{0}}\right)\right]\right\},
$$

which is shown in Fig. 2, along with the Fermi-liquid result. Note that near $T \approx 2 T_{0}$, we find that $G_{A B}$ displays a pronounced maximum, also in striking contrast to a Fermi liquid.

Increasing the temperature further we cross over into the $V \ll T_{0} \ll T$ regime where $G_{0}$ scales as in (32), but

$$
G_{\mathrm{AB}} \propto\left(\frac{T}{T_{0}}\right)\left(\frac{T}{T_{\mathrm{F}}}\right)^{2 q-2} e^{-q T / T_{0}} .
$$

Thus $G_{A B}$ exhibits a crossover from the well-known $T^{2 q-2}$ Luttinger liquid behavior to a new scaling behavior which is much closer to a Fermi liquid $(q=1)$. Careful measurements in this experimentally accessible regime should be able to distinguish between a Fermi liquid and this predicted nearly Fermi-liquid temperature dependence. 


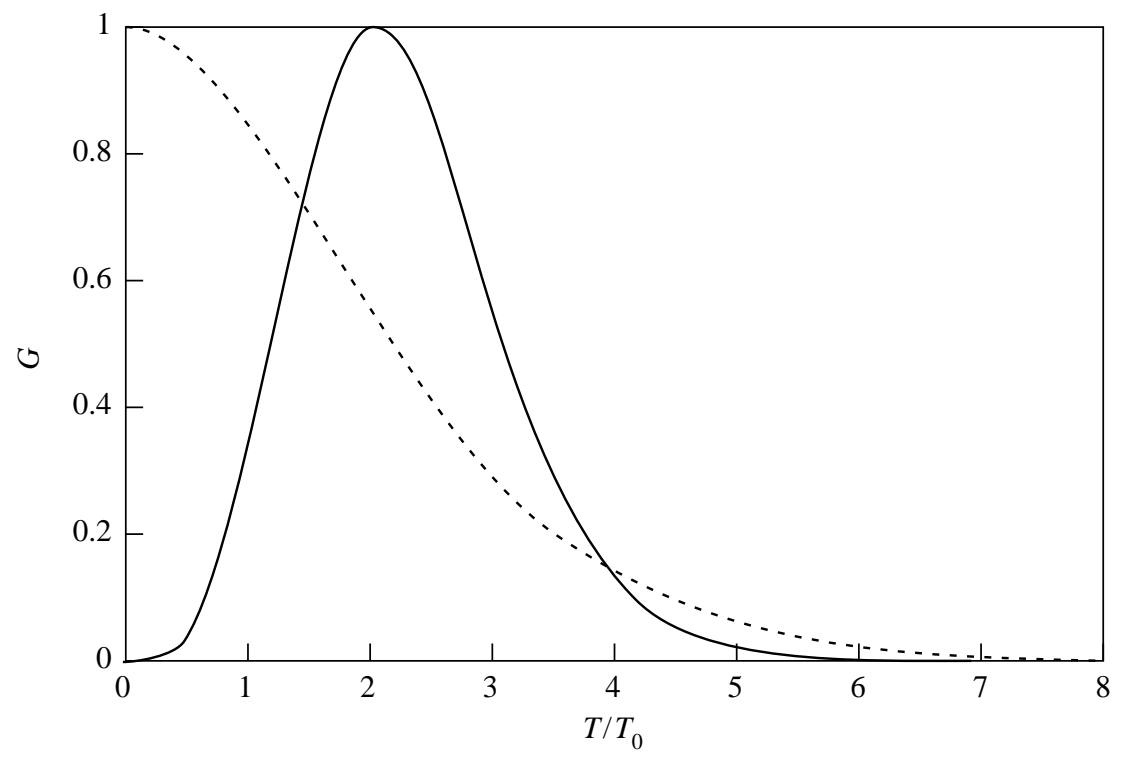

Fig. 2. Temperature dependence of $G_{\mathrm{AB}}$ for the cases $g=1$ (dashed curve) and $g=1 / 3$ (solid curve). Both curves are normalized to have unit amplitude at their respective maxima.

\subsection{High voltage regime: $V \gg T_{0}$}

Again there are three temperature regimes. For the lowest temperatures $T \ll T_{0} \ll V$, the response is again nonlinear. The direct term varies with voltage as $I_{0} \propto V^{2 q-1}$, as in the lowest temperature, low voltage regime. However, the $\mathrm{AB}$ curent is now much more complicated, involving power-laws times trigonometric functions of the ratio $V / T_{0}$. As an example, for the case $q=3$ we find

$$
I_{\mathrm{AB}} \propto-\left[\frac{3 V}{2 \pi T_{0}}\right] \cos \left(\frac{V}{2 \pi T_{0}}\right)+\left[3-\left(\frac{V}{2 \pi T_{0}}\right)^{2}\right] \sin \left(\frac{V}{2 \pi T_{0}}\right),
$$

which is related to the Bessel function $J_{5 / 2}$.

As the temperature is increased further to $T_{0} \ll T \ll V$, we find a crossover to a remarkable hightemperature nonlinear regime. Here $I_{0} \propto V^{2 q-1}$ as before, but now

$$
I_{\mathrm{AB}} \propto\left(\frac{T}{T_{0}}\right)^{q} e^{-q T / T_{0}} V^{q-1} \sin \left(\frac{V}{2 \pi T_{0}}\right) .
$$

Therefore, the nonlinear response at fixed temperature can also be used to distinguish between Fermi liquid and Luttinger liquid behavior, even at relatively high temperatures.

When the temperature exceeds $\mathrm{V}$, the response finally becomes linear. When $T_{0} \ll V \ll T, G_{0}$ scales as in (32) whereas $G_{A B}$ scales as in (34). Thus at high temperatures the low- and high-voltage regimes behave similarily.

\section{Persistent current in a chiral Luttinger liquid}

In the previous section we have been discussing the transport properties of an edge state that occurs at 
the boundary of a quantum Hall fluid pierced by an antidot potential. In this section we shall discuss a nonFermi-liquid mesoscopic property of the edge current occurring at this same type of antidot boundary or at the boundary of a FQHE droplet confined in a quantum dot.

In a macroscopic edge state, an equilibrium edge current exists even in the absence of additional $\mathrm{AB}$ flux or twisted boundary conditions. In the absence of disorder the magnitude of this current is universal and is given by [32]

$$
I_{e d g e}=g \frac{\omega_{\mathrm{c}}}{4 \pi}+\frac{\tilde{\epsilon}_{q h}}{2 \pi}
$$

where $\omega_{c}$ is the cyclotron frequency and $\tilde{\epsilon}_{q h}$ is the proper quasihole energy of the Laughlin state at filling factor $g=1 / q$.

We now couple the edge state to an additional AB flux $\Phi$. The grand-canonical partition function of the mesoscopic edge state factorizes into a zero-mode contribution,

$$
Z^{0}=\sum_{n=-\infty}^{\infty} e^{-g \pi^{2}\left(T_{0} / T\right)\left(n-\Phi / \Phi_{0}\right)^{2}},
$$

which depends on $\Phi$, and an irrelevant flux-independent contribution from the nonzero-modes. Here $T_{0}$ is again given by eqn (25). Note that if $N_{ \pm}$were restricted to be an integer then the period of these equilibrium AB oscillations would be $\Phi_{0} / g$. The allowed fractionally charged excitations (18) are therefore responsible for restoring the $\mathrm{AB}$ period to $\Phi_{0}$, as is well-known in other contexts [33, 34].

The edge current induced from the additional flux $\Phi$ is

$$
I \equiv-\frac{\partial \Omega}{\partial \Phi}=\frac{2 \pi T}{\Phi_{0}} \sum_{n=1}^{\infty}(-1)^{n} \frac{\sin \left(2 \pi n \Phi / \Phi_{0}\right)}{\sinh \left(n q T / T_{0}\right)},
$$

where $\Omega$ is the grand-canonical potential. At zero temperature, this chiral persistent current has an amplitude (with units now restored)

$$
\bar{I}=g \frac{e v}{L},
$$

where, again, $L$ is the length of the edge state, and $\bar{I}$ is renormalized by the electron-electron interactions in precisely the same way as in a nonchiral Luttinger liquid [28]. At temperatures $T \gg T_{0}$ the amplitude decays as $\sim e^{-q T / T_{0}}$. Because these persistent currents are chiral, there is no backscattering from impurities and hence no amplitude reduction from disorder. The temperature dependence of the magnetic response of a FQHE edge state may therefore be an ideal system to observe non-Fermi-liquid behavior.

\section{Conclusion}

We have studied the $\mathrm{AB}$ effect for filling factor $1 / q$ ( $q$ odd) in the strong-antidot-coupling limit with CLL theory. The low-temperature linear response is similar to that in a quantum point contact. However, the $\mathrm{AB}$ oscillations are a mesoscopic effect and, as such, are diminished in amplitude above a crossover temperature $T_{0}$ determined by the size of the antidot. Above $T_{0}$, the temperature dependence of the $\mathrm{AB}$ oscillations is qualitatively similar to that in a Fermi liquid (see Fig. 2). It is clear that a related crossover occurs in the weak-antidot-coupling regime as well. In addition, we have identified a new high-temperature nonlinear response regime that may also be used to distinguish between a Fermi and Luttinger liquid. Finally, we have predicted new mesoscopic edge-current oscillations that have a universal non-Fermi-liquid temperature dependence and may be another means to observe a chiral Luttinger liquid.

Acknowledgements - This work has been supported by NSERC of Canada. 


\section{References}

[1] K. von Klitzing, G. Dorda and M. Pepper, Phys. Rev. Lett. 45, 494 (1980).

[2] See S. E. Ulloa, A. MacKinnon, E. Castano and G. Kirczenow, in Handbook on Semiconductors, Vol.1, Edited by P. T. Landsberg, North Holland (1992), for a review.

[3] B. I. Halperin, Phys. Rev. B 25, 2185 (1982).

[4] M. Büttiker, Phys. Rev. Lett. 57, 1761 (1986); P. Streda, J. Kucera and A. H. MacDonald, Phys. Rev. Lett. 59,1973(1987); J. K. Jain and S. A. Kivelson, Phys. Rev. Lett. 60,1542 (1988); M. Büttiker, Phys. Rev. B 38, 9375 (1988).

[5] R. B. Laughlin, Phys. Rev. Lett. 50, 1395 (1983).

[6] D. C. Tsui, H. L. Stormer and A. C. Gossard, Phys. Rev. Lett. 48, 1559 (1982).

[7] X. G. Wen, Phys. Rev. B 41, 12838 (1990); Phys. Rev. B 43, 11025 (1991).

[8] K. Moon, H. Yi, C. L. Kane, S. M. Girvin and M. P. A. Fisher, Phys. Rev. Lett. 71, 4381 (1993).

[9] V. L. Pokrovsky and L. P. Pryadko, Phys. Rev. Lett. 72, 124 (1994).

[10] C. L. Kane, M. P. A. Fisher and J. Polchinski, Phys. Rev. Lett. 72, 4129 (1994). C. L. Kane and M. P. A. Fisher, Phys. Rev. B 51, 13449 (1995); Phys. Rev. B 52, 17393 (1995).

[11] P. Fendley, A. W. W. Ludwig and H. Saleur, Phys. Rev. Lett. 74, 3005 (1995); Phys. Rev. B 52, 8934 (1995).

[12] J. K. Jain, Phys. Rev. Lett. 63, 199 (1989); Science 266, 1199 (1994).

[13] G. Kirczenow and B. L. Johnson, Phys. Rev. B 51, 17579 (1995); see cond-mat/950258 at world wide web site http://xxx. lanl.gov/ for figures for this paper. G. Kirczenow and B. L. Johnson, Surf. Science (in press).

[14] F. P. Milliken, C. P. Umbach and R. A. Webb, Solid St. Comm. 97, 309 (1996).

[15] J. D. F. Franklin, I. Zailer, C. J. B. Ford, P. J. Simpson, J. E. F. Frost, D. A. Ritchie, M. Y. Simmons and M. Pepper, Surf. Science (in press).

[16] I. J. Maasilta and V. J. Goldman (preprint).

[17] A. M. Chang, L. N. Pfeiffer, and K. W. West (preprint).

[18] We define resonance here in the sense it is used in the Luttinger liquid literature: The system is said to be on resonance when the $\mathrm{AB}$ flux prevents transmission through the antidot, i.e., if $\Phi=(n+1 / 2) \Phi_{0}$, where $n$ is integral. Thus, CLL theory at low temperature predicts peaks in the two-terminal conductance on resonance, and vanishing conductance elsewhere. This definition is opposite to what is usually used in the FL antidot literature, which defines resonance to occur when $\Omega=2 \pi n$ in eqn (3), which means that for an isolated antidot the Fermi level coincides with an energy level of the antidot.

[19] P. J. Simpson, et al., Surf. Sci. 305, 453 (1994); G. Kirczenow, et al., Phys. Rev. Lett. 72, 2069 (1994); A. S. Sachrajda et al., Phys. Rev. B 50, 10856 (1994).

[20] G. Kirczenow, Phys. Rev. B 50,1649 (1994).

[21] G. Kirczenow, Phys. Rev. B (to be published).

[22] A. Lopez and E. Fradkin, Phys. Rev. B 44, 5246 (1991).

[23] B. I. Halperin, P. A. Lee and N. Read, Phys. Rev. B 47, 7312 (1993).

[24] S. C. Zhang, H. Hansson and S. A. Kivelson, Phys. Rev. Lett. 62, 82 (1989); B. Rejaei and C. W. J. Beenakker, Phys. Rev. B 43, 11392 (1991); Phys. Rev. B 46, 15566 (1992). S. C. Zhang, Int. J. Mod. Phys. B 6, 25 (1992). A. S. Goldhaber and J. K. Jain, Phys. Lett. A 199, 267 (1995).

[25] A somewhat different result for zero temperature has been given by L. Brey, Phys. Rev. B 50, 11861 (1994)

[26] Private communication with J. D. F. Franklin.

[27] F. D. M. Haldane, J. Phys. C 14, 2585 (1981).

[28] D. Loss, Phys. Rev. Lett. 69, 343 (1992).

[29] C. L. Kane and M. P. A. Fisher, Phys. Rev. Lett. 68, 1220 (1992); Phys. Rev. B 46, 15233 (1992). 
[30] X. G. Wen, Phys. Rev. B 44, 5708 (1991).

[31] To simplify our expressions in the nonlinear response regime we apply the potential difference $V$ symmetrically about an antidot energy level, which allows there to be a finite linear response at zero temperature in the FL case. Therefore, as the voltage is varied the two chemical potentials move in opposite directions at half the rate at which the voltage changes. A consequence of this symmetrization is that the nonlinear response in the FL case has a simple sinusoidal dependence on $V$, with a period equal to twice the antidot level spacing. For general situations when $\mu_{d}=\mu_{s}-V$ (but $\mu_{s}$ arbitrary), the FL result is instead $I^{F L}=|t|^{2}\left\{V+\frac{2 \pi k_{B} T}{\sinh \left(T / T_{0}\right)} \sin \left(\frac{V L}{2 v}\right) \cos \left[\frac{L}{2 v}\left(V-2 \mu_{s}\right)-2 \pi \Phi / \Phi_{0}\right]\right\}$. The special symmetric case above is recovered for $\mu_{s}=V / 2$. For the choice $\mu_{s}=0$ the period is equal to the level spacing.

[32] M. R. Geller and G. Vignale, Phys. Rev. B 52, 14137 (1995).

[33] P. A. Lee, Phys. Rev. Lett. 65, 2206 (1990).

[34] D. J. Thouless and Y. Gefen, Phys. Rev. Lett. 66, 806 (1991). 